\title{
Comparative study of capillary blood glucose estimation by glucometer and venous plasma glucose estimation in women undergoing the one step DIPSI test (diabetes in pregnancy study group India) for screening and diagnosis of gestational diabetes mellitus
}

\author{
Deepali S. Jadhav*, Uma N. Wankhede
}

Department of Obstetrics and Gynecology, B.J.G.M.C. and S.G.H. Pune, Maharashtra, India

Received: 30 January 2017

Accepted: 03 March 2017

\section{*Correspondence:}

Dr. Deepali S Jadhav,

E-mail: deepjadhav1988@gmail.com

Copyright: ( $)$ the author(s), publisher and licensee Medip Academy. This is an open-access article distributed under the terms of the Creative Commons Attribution Non-Commercial License, which permits unrestricted non-commercial use, distribution, and reproduction in any medium, provided the original work is properly cited.

\begin{abstract}
Background: Gestational diabetes mellitus (GDM) is defined as carbohydrate intolerance of variable severity with onset or first recognition during pregnancy. The importance of GDM is that two generations are at risk of developing diabetes in the future. Aim was to study the merits and demerits of capillary blood glucose estimation by glucometer over venous plasma glucose estimation while performing DIPSI test.

Methods: It was a hospital based clinical study. 1000 patients were enrolled between 24-28 weeks of gestation and DIPSI test was performed. Patient was instructed to come irrespective of fasting. $75 \mathrm{~g}$ glucose dissolved in 200-400 $\mathrm{ml}$ of water and patient was asked to drink in 5 minutes. Venous blood was drawn after 2 hours, capillary blood sugar also was measured at the same time by glucometer.

Results: Sensitivity of capillary blood sugar (CBS) method in detecting GDM is 100\% as compared to venous plasma glucose (VPG) and specificity is $99.46 \%$ as compared to VPG. Considering the agreement between two methods for diagnosis of GDM, equal sensitivity of both methods and small number of false positive cases detected by CBS method, due to almost equal specificity $(99.46 \%)$, CBS method by glucometer can be recommended as an alternative to VPG method as a screening and diagnostic test for GDM.

Conclusions: It is appropriate and feasible to offer capillary blood sugar sampling by DIPSI test for screening and diagnosis of GDM. The prevalence of GDM in our study is $8 \%$ by capillary blood sugar sampling and $7.5 \%$ by venous plasma glucose sampling according to DIPSI test.
\end{abstract}

Keywords: Capillary blood sugar, DIPSI, GDM

\section{INTRODUCTION}

Gestational diabetes mellitus is defined as carbohydrate intolerance of variable severity with onset or first recognition during pregnancy. ${ }^{1}$ Importance of GDM is that two generations are at risk of developing diabetes in the future. Women with history of GDM are at increased risk of future diabetes, predominantly type 2 diabetes, as their children. ${ }^{2}$ Thus GDM offers an important opportunity for diagnosis and implementation of clinical strategies for diabetes prevention. Timely action taken now in screening in all pregnant women for glucose intolerance, achieving euglycemia in them and ensuring adequate nutrition may prevent in all probability, the vicious cycle of transmitting glucose intolerance from one generation to another. However, for the detection and diagnosis of GDM, controversy concerning optimal strategy still continues. 
The American diabetes association (ADA) recommends two step procedures for screening and diagnosis of diabetes in selective population. ${ }^{3}$ Compared with selective screening, universal screening for GDM detects more cases and improves maternal offspring prognosis. ${ }^{4}$ In the Indian context, screening is essential in all pregnant women as the Indian women have an eleven fold increased risk of developing glucose intolerance during pregnancy compared to Caucasian women. ${ }^{5}$

Another area of concern is that among ethnic groups in South Asian countries, the Indian women have the highest frequency of GDM. The recent data shows $16.55 \%$ of prevalence of GDM in our country. ${ }^{6}$ Hence, universal screening has become important in our country. For this we need a simple procedure which should be economical and feasible. Hence an experimental comparative study is being conducted to find out a onestep procedure which can serve both as screening and diagnostic tool at the same time and which is acceptable, economical and feasible to perform in the Indian context.

\section{METHODS}

It was a hospital based study. 1000 pregnant women in second trimester between 24-28 weeks of gestational age who attend antenatal clinic of tertiary care center in a time period of one year (September 2015 to August 2016) were enrolled in this study after providing informed consent. A hospital based clinical study designed to compare the merits and demerits of venous plasma glucose estimation and capillary blood glucose estimation by glucometer after DIPSI test i.e. 2 hour blood glucose test post $75 \mathrm{~g}$ glucose challenge irrespective of fasting state.

A detailed assessment of patient was performed including history (any family history of diabetes, history of previous pregnancies and socioeconomic status etc.), general examination and obstetric examination. Routine investigations during antenatal visits were done. Informed consent to participation was taken during initial assessment.

A standard form was used to record the data of tests performed, detailed clinical assessment of patients, including history and examination findings, investigations including the test results.

\section{Method of performing 2 hour 75 g oral glucose tolerance test (DIPSI)}

Patient was instructed to come irrespective of fasting, was asked to take a carbohydrate unrestricted diet (not less than $150 \mathrm{~g}$ per day) for 3 days before test was performed. $75 \mathrm{~g}$ of glucose was dissolved in 200-400 ml of water and was asked to drink in 5 minutes. Venous blood drawn after 2 hours and capillary blood sugar was also measured at the same time by finger prick method using glucometer. Patient was seating while taking sample. ${ }^{7}$

Diabetes in pregnancy study group India (DIPSI) diagnostic criterion of 2-h plasma glucose $\geq 7.8 \mathrm{mmol} / \mathrm{L}$ with $75 \mathrm{~g}$ oral glucose load is a modified version of WHO criterion, in that the WHO procedure requires women to be in the fasting state, whereas DIPSI procedure was performed in the fasting/ non fasting state irrespective of last meal timing. This prospective had been undertaken to ascertain the validity of DIPSI criterion by capillary blood glucose estimation by glucometer to diagnose GDM based on pregnancy outcome in Indian population.

The plasma glucose was estimated in automated analyzer in central lab of S.G.H. A specific reagent was used for glucose determination in the analyzer. It was performed by GOD-POD methodology.

\section{Principle}

Enzymatic colorimetric determination of glucose according to the following reaction:

Glucose $+\mathrm{O}_{2} \rightarrow$ Gluconic acid $+\mathrm{H}_{2} \mathrm{O}_{2}$ (after the action of glucose oxidase)

$2 \mathrm{H}_{2} \mathrm{O}_{2}+$ Phenol + 4-Aminoantipyrine $\rightarrow$ Quinonimine + $4 \mathrm{H}_{2} \mathrm{O}$

Table 1: Diagnosis of GDM according to DIPSI criteria

\begin{tabular}{|ll|}
\hline Interpretation of OGTT & $\begin{array}{l}2 \text { hour venous blood } \\
\text { sugar (mg/dl) }\end{array}$ \\
\hline Normal & $<140$ \\
\hline Gestational diabetes mellitus & $140-199$ \\
\hline Overt diabetes & $\geq 200$ \\
\hline
\end{tabular}

\section{Inclusion criteria}

- All consenting pregnant women in second trimester between 24-28 weeks.

- Pregnant women of any parity.

- $\quad$ Singleton pregnancy.

\section{Exclusion criteria}

- Pre-gestational diabetes.

- Chronic renal/ cardiac/ hepatic/ respiratory diseases.

- Taking drugs that alter glucose metabolism.

- Women who refuse to participate.

\section{RESULTS}

Out of 1000 women screened by oral glucose tolerance test, 80 women were found to have gestational diabetes mellitus. 
Hence, prevalence of gestational diabetes mellitus was $8 \%$ as per capillary blood sugar sampling by DIPSI test (Table 2). Prevalence of gestational diabetes mellitus.

Table 2: Prevalence of GDM by CBS sampling method.

\begin{tabular}{|l|l|l|}
\hline $\begin{array}{l}\text { Number of } \\
\text { women screened }\end{array}$ & $\begin{array}{l}\text { Number of } \\
\text { women with GDM }\end{array}$ & Percent \\
\hline 1000 & 80 & 8 \\
\hline
\end{tabular}

According to the Table 3, out of 1000 women screened by oral glucose tolerance test, 75 were found to have gestational diabetes mellitus. Hence prevalence of GDM was $7.5 \%$ as per venous plasma glucose sampling by DIPSI test.

Table 3: Prevalence of GDM by venous plasma glucose sampling method.

\begin{tabular}{|lll|}
\hline $\begin{array}{l}\text { Number of } \\
\text { women screened }\end{array}$ & $\begin{array}{l}\text { Number of women } \\
\text { with GDM }\end{array}$ & Percent \\
\hline 1000 & 75 & 7.5 \\
\hline
\end{tabular}

Table 4: Comparison of gestational diabetes mellitus as per capillary blood sugar and venous plasma glucose sampling by DIPSI test.

\begin{tabular}{|lllll|}
\hline \multicolumn{1}{|l}{ Test result } & \multicolumn{2}{l|}{ Capillary blood sugar levels } \\
\hline \multirow{2}{*}{ Venous plasma glucose level } & Yes & No & Total \\
\cline { 2 - 5 } & Yes & $75(93.75 \%)$ & 0 & $75(7.5 \%)$ \\
\hline Total & & $5(6.25 \%)$ & $920(100 \%)$ & $925(92.5 \%)$ \\
\hline
\end{tabular}

According to Table 4, out of 1000 women screened by oral glucose tolerance test, $80(8 \%)$ women were found to have GDM by venous plasma glucose sampling and 75 $(7.5 \%)$ women were found to have GDM by capillary blood sugar sampling as per DIPSI criterion. Additionally $5(6.75 \%)$ women were diagnosed with GDM by capillary blood sugar sampling.

In comparison of both tests, it was found that overall agreement in $99.5 \%$ of cases with Cohen's kappa (k) Index value is 0.964 (fair agreement) for diagnosis of GDM.

$$
\begin{gathered}
\text { Sensitivity }=\frac{\text { True Positive }}{\text { True Positive }+ \text { False Negative }} \\
=\frac{75}{75+0}=100 \% \\
\text { Specificity }=\frac{\text { True Negative }}{\text { True Negative + False Positive }} \\
=\frac{925}{925+5}=99.46 \%
\end{gathered}
$$

With comparison to venous plasma glucose sampling, sensitivity of capillary blood glucose sampling by DIPSI criterion is $100 \%$ and specificity is $99.46 \%$.

\section{DISCUSSION}

Present study was a prospective, clinical, observational study, was conducted in tertiary care center Pune, India which was first of its kind to be undertaken evaluating capillary blood sugar estimation by glucometer for screening and diagnosis of GDM using DIPSI criteria. This study involved screening of total 1000 patients registered with us. Patients were screened at the time of their antenatal visit between 24-28 weeks gestational age. This time window was selected because the insulin resistance that causes hyperglycemia increases as the third trimester progresses, early testing may miss some patients who later become glucose intolerant. Performing the test too late in third trimester limits the time in which metabolic intervention can take place. For this reason it is recommended that glucose tolerance test be performed in all pregnant women at 24-28 weeks gestation. ${ }^{8}$

Aim of the study was to compare merits and demerits of capillary blood glucose estimation by glucometer over venous plasma glucose estimation by DIPSI test, to study the incidence of GDM in our study.

Comparison of diagnosis of GDM by capillary blood sugar (CBS) estimation by glucometer with venous plasma glucose (VPG) estimation as per DIPSI test

Sensitivity of CBS method in detecting GDM was $100 \%$ compared to VPG, and specificity of CBS was $99.46 \%$ as compared to VPG. In comparison of both tests it was found that overall agreement in $99.5 \%$ of cases with Cohen's kappa ( $\kappa)$ Index value is 0.965 (fair agreement) for diagnosis of gestational diabetes mellitus.

Considering the fair agreement between two methods for diagnosis of gestational diabetes mellitus, equal sensitivity of both methods and small number of false 
positive cases detected by CBS method, due to almost equal specificity $(99.46 \%)$, CBS method by glucometer can be recommended as an alternative to VPG method as a screening and diagnostic test for gestational diabetes mellitus.

\section{Advantages of VPG estimation method}

- Recommended as standard method for screening for GDM by renowned agencies like ADA, WHO etc. ${ }^{9}$

- Values reflect actual glucose level in body which is essential in tight glycemic control situation like neonatal sugar monitoring, gestational diabetes.

- No question of calibration arises in VPG method, as it is done in laboratory setup.

\section{Limitations of VPG estimation method}

- No portability possible.

- Cannot be done on OPD basis, as it has to be accompanied by biochemical laboratory facility.

- Time consuming procedure.

- Expensive.

\section{Limitations of glucometer method}

Discrepancy between simultaneously collected VPG and CBG due to physiological phenomenon. Whole blood (e.g. a capillary sample) is composed predominantly of plasma and cells. In the laboratory, glucose is measured on a plasma sample i.e. a whole blood sample is centrifuged, followed by removal of the cellular component of blood. Red cells have a lower water and glucose content than plasma. As a result of this, the glucose concentration of whole blood is about $11 \%$ less than the glucose concentration of plasma. A second major difference between capillary and laboratory venous results, relates to the fact that the glucose value of a capillary sample is higher than for a corresponding venous sample, because glucose uptake by tissues as blood flows from the capillaries to the veins partially depletes the venous sample of glucose. Tissue uptake of glucose increases after food. The glucose gradient between capillary and venous samples therefore shows a postprandial increase which may be as high as $20 \%$ total glucose concentration.

Problem of calibration may be present with some type of glucometers but one touch glucometers (as used in present study) don't require any calibration codes while operating. Contamination of test finger surface with contaminants like glucose rich foodstuff which may elevate the value) or water (inadequate hand drying after washing) may produce a dilutional error. Strip performance is likely to be sub optimal, once strip passes the expiry date. Performance may also be affected by prolonged exposure to adverse environmental conditions like heat, humidity. Pathophysiological factors present in patient like hypoxia (including acrocyanosis), extremes of hematocrit like anaemia, hyperviscosity syndromes, sufficient to affect accuracy of glucose readings.

\section{Advantages of glucometer method}

- Portable, convenient yet inexpensive.

- $\quad$ Short testing time. Test results are analyzed almost instantly.

- Blood sample is easy to obtain. There are several collection sites on the body and these can be rotated.

- Testing can be done at OPD and with little training

- Good sensitivity and specificity.

- Economical: As no laboratory setup is required to carry out biochemical investigations from treatment providers' point of view and cheap glucometer strips make it economically viable from patients' point of view. Glucometer analytical performance and ease of use has improved markedly over recent years. An understanding of glucose physiology and glucometer performance should help minimize glucometer related errors and help with trouble shooting. VPG estimation method in laboratory has remained the cornerstone procedure in diagnosis of gestational diabetes and has been granted worldwide recognition. Considering the high prevalence of GDM in Indian population, universal screening approach has become essential. So the test which is simple, quick, reliable, convenient, feasible, economical, compliance generating is the need of time. CBG estimation by glucometer fulfills all the above mentioned criteria and can be effectively recommended as the screening and diagnostic tool for gestational diabetes mellitus. ${ }^{10}$

\section{CONCLUSION}

It is appropriate and feasible to offer capillary blood sugar sampling by DIPSI test for screening and diagnosis of GDM. Prevalence of GDM in our study population is $8 \%$ by capillary blood sugar sampling and $7.5 \%$ by venous blood glucose sampling according to DIPSI test.

\section{Funding: No funding sources \\ Conflict of interest: None declared \\ Ethical approval: Not required}

\section{REFERENCES}

1. Committee on Obstetric Practice. Committee opinion no. 504: screening and diagnosis of gestational diabetes mellitus. Obstet Gynecol. 2011;118(3):751-3.

2. American diabetic association. Gestational diabetes mellitus. Diabetes Care. 2004;27(1):88-90.

3. American Diabetes Association. Standards of medical care in diabetes. Diabetes Care. 2004;27(1):15-35.

4. Moses RG, Cheung NW. Point: universal screening for gestational diabetes mellitus. Diabetes Care. 2009;32(7):1349-51. 
5. Wahi P, Dogra V, Jandial K, Bhagat R, Gupta R, Gupta S, et al. Prevalence of gestational diabetes mellitus (GDM) and its outcomes in Jammu region. $\mathbf{J}$ Assoc Physicians India. 2011;59(4):227-30.

6. Sharma K, Wahi P, Gupta A, Jandial K, Bhagat R, Gupta $R$ et al. Single glucose challenge test procedure for diagnosis of gestational diabetes mellitus: a Jammu cohort study. J Assoc Physicians India. 2013;61(8):558-9.

7. Seshiah V, Balaji V, Balaji MS, Sekar A, Sanjeevi $\mathrm{CB}$, Green A. One step procedure for screening and diagnosis of gestational diabetes mellitus. Diabetes. 2005;126:200.

8. Magon N. Gestational diabetes mellitus: Get, set, go from diabetes capital of the world to diabetes care capital of the world. Indian $\mathbf{J}$ Endocrinol. Metabolism. 2011;15(3):161.

9. Sacks DA, Chen W, Tsadik G, Buchanan TA. Fasting plasma glucose test at the first prenatal visit as a screen for gestational diabetes. Obst Gynecol. 2003;101(6):1197-203.

10. Hawkins JS, Casey BM, Lo JY, Moss K, McIntire DD, Leveno KJ. Weekly compared with daily blood glucose monitoring in women with diet-treated gestational diabetes. Obst Gynecol. 2009;113(6):1307-12.

Cite this article as: Jadhav DS, Wankhede UN. Comparative study of capillary blood glucose estimation by glucometer and venous plasma glucose estimation in women undergoing the one step DIPSI test (diabetes in pregnancy study group India) for screening and diagnosis of gestational diabetes mellitus. Int J Reprod Contracept Obstet Gynecol 2017;6:1488-92. 